\title{
Bei Kindern oft nicht (rechtzeitig) erkannt
}

Große Unsicherheiten bestehen bei Diagnostik und Therapie eines Schlaganfalls im Kindesalter. Nur bei jedem dritten Kind wurde einer schwedischen Studie zufolge die Diagnose innerhalb von sechs Stunden gestellt. Die Inzidenz lag bei 1,8 pro 100000 Kindern und Jahr. Das jüngste Kind war erst sieben Monate. Innerhalb von sieben Jahren verstarben $8 \%$ der Kin- der. $85 \%$ der Überlebenden trugen Langzeitschäden davon. Ein Drittel hatte bereits Vorerkrankungen, ein weiteres Drittel Gefäßanomalien. Als wichtiger Risikofaktor ergab sich bei weiblichen Teenagern eine Kombination aus der Einnahme eines oralen Kontrazeptivums verbunden mit Rauchen und einer Eisenmangelanämie. Acta Paediatrica 2010;99:1641-1649 und 1650-1656

\section{VENÖSE THROMBOEMBOLIE}

\section{Wie hoch ist das Rezidivrisiko?}

Die Wahrscheinlichkeit, dass ein Patient zum zweiten Mal eine venöse Thromboembolie (VTE) erleidet, hängt entscheidend vom Auslöser des Erstereignisses ab. Wie hoch das jeweilige Risiko ist, wurde in einer systematischen Übersichtsarbeit ermittelt. Alle Patienten waren nach der ersten VTE mindestens drei Monate antikoaguliert worden. Das Ergebnis: 24 Monate nach Therapieende lag die Wiederholungsrate bei $3,3 \%$ pro Patientenjahr bei Vorliegen eines transienten Risikofaktors. Handelte es sich dabei um eine Operation, betrug die Rate nur $0,7 \%$. Deutlich häufiger war eine erneute VTE bei einer idiopathischen ersten VTE - mit 7,4\% pro Patientenjahr. Arch Intern Med 2010;170(19):1710-1716

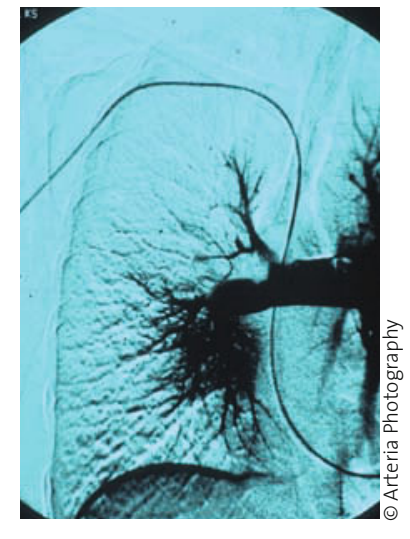

Lungenembolie.

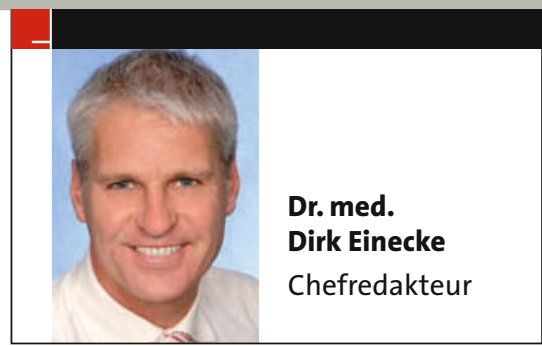

\section{STATIONÄRE BEHANDLUNG}

\section{Danach geht es mit Senioren oft bergab}

Alte Menschen, die bisher im Alltag selbstständig zurecht kamen, werden häufig dauerhaft hilfsbedürftig, wenn sie im Krankenhaus behandelt werden müssen. Auch krankheitsbedingte Immobilität führt oft zu bleibenden Einschränkungen bei Alltagsaktivitäten wie sich waschen, sich ankleiden oder gehen. Besonders verhängnisvoll sind sturzbedingte Verletzungen, und besonders gefährdet sind Senioren, die ohnehin schon gebrechlich sind. Mit diesen Ergebnissen bestätigt eine prospektive 10-Jahres-Studie mit 754 über 70-Jährigen das, was viele Ärzte aus ihrem Praxisalltag kennen. JAMA 2010;304(17):1919-1928

\section{ARBEITSMEDIZIN}

\section{Mehr Unfälle bei Schichtarbeiterinnen}

Personen, die in wechselnden Schichten oder nachts arbeiten, haben ein fast doppelt so hohes Unfallsrisiko wie Personen mit regelmäßigen Arbeitszeiten am Tage. Besonders gefährdet sind Frauen. Hier ist die Risiko von Arbeitsunfällen bei Schichtund Nachtarbeit um 95\% höher. Bei Männer ist das Unfallrisiko um 50\% erhöht. Der Unterschied erklärt sich vermutlich dadurch, dass sich die Frauen meist auch noch um den Haushalt und die Kinder kümmern und dadurch noch gravierendere Schlafdefizite haben.

Scandinavian Journal of Work, Environment and Health, 2010 